\title{
THE SPES3 FACILITY FOR TESTING AN INTEGRAL LAYOUT SMR: BDBE SIMULATION ANALYSIS
}

\author{
Roberta Ferri \\ SIET S.p.A. \\ Piacenza, Italy
Fosco Bianchi
ENEA \\ Bologna, Italy
}

\author{
Andrea Achilli \\ SIET S.p.A. \\ Piacenza, Italy
}
Paride Meloni
ENEA
Bologna, Italy

\author{
Cinzia Congiu \\ SIET S.p.A. \\ Piacenza, Italy
}
Stefano Monti
ENEA
Bologna, Italy

\author{
Gustavo Cattadori \\ SIET S.p.A. \\ Piacenza, Italy
}
Alfredo Luce
ENEA
Saluggia, Italy

\author{
Marco Enrico Ricotti \\ Davide Papini \\ Milano, Italy
}

\author{
Davor Grgić \\ University of Zagreb. FER \\ Zagreb, Croatia
}

\begin{abstract}
The SPES3 facility is being built at the SIET laboratories, in the frame of an R\&D program on Nuclear Fission, led by ENEA and funded by the Italian Ministry of Economic Development. The facility is based on the IRIS reactor design, an advanced medium size, integral layout, pressurized water reactor, based on the proven technology of PWR with an innovative configuration and safety features suitable to cope with Loss of Coolant Accidents through a dynamic coupling of the primary and containment systems. SPES3 is suitable to test the plant response to postulated Design and Beyond Design Basis Events, providing experimental data for code validation and plant safety analysis. It reproduces the primary, secondary and containment systems of the reactor with 1:100 volume scale, full elevation, prototypical fluid and thermal-hydraulic conditions. A design-calculation feedback process, based on the comparison between IRIS and SPES3 simulations, performed respectively by FER, with GOTHIC and RELAP5 coupled codes, and by SIET, with RELAP5 code, led to reduce the differences in the two plants behaviour, versus a 2-inch equivalent DVI line DEG break, considered the most challenging LOCA for the IRIS plant. Once available the final design of SPES3, further calculations were performed to investigate Beyond Design Basis Events, where the intervention of the Passive Containment Condenser is fundamental for the accident recovery. Sensitivity analyses showed the importance of the PCC actuation time, to limit the
\end{abstract}

containment pressure, to reach an early pressure equalization between the primary and containment systems and to allow passive water transfer from the containment to the RPV, enhanced by the ADS Stage-II opening.

\section{INTRODUCTION}

One of the most fascinating promises of the nuclear renaissance set its bases on the Small and medium Modular Reactor development. The continuous increase of the worldwide power demand, the need of energy production in remote areas and the need of public acceptance of new nuclear installations pushes toward simplicity, advanced safety, economics and fast construction of new Nuclear Power Plants.

The integral layout SMRs offer technological advantages as the reduction of the penetration number on the RPV, the piping size reduction with the possibility of only Small Break LOCAs, the reduction of power and source term with possible elimination of the EPZ, the suitability for non electric applications as desalination and process heat, the innovative design and fuel cycle to proliferation resistance. Moreover, given the different layout by the traditional NPPs, new concept of I\&C technology is developed to allow in-vessel measurements for diagnostic, control and plant operation.

The IRIS reactor has been developed for about ten years by the IRIS consortium (utilities, industries, research centers and universities), reaching an advanced design status. With its integral design, it represents an advanced engineering solution 
of the latest proven LWR technology. Pressurized, medium size, safe, modular and economic, it provides a viable bridge to Generation IV and satisfies the GNEP requirements for gridappropriate NPPs $(1,2)$.

The need to verify the whole plant behavior on an Integral Test Facility, suitable to simulate postulated accidental transients, led ENEA, member of the consortium, to take in charge of the ITF experimental program. On the basis of the over thirty-years experience on thermal-hydraulic test facilities, the SIET company was chosen to design, build and operate the SPES3 facility.

SPES3, even if IRIS design oriented, is anyway suitable to general interest studies on thermal-hydraulic phenomena like primary to containment dynamic coupling, two-phase flow measurements, system interaction and to code qualification for SMR simulation. It will provide experience, data and information aimed at increasing the SMR safety, independent by the different vendors SMR designs.

The design of SPES3 was carried out according to the following steps:

- preliminary design based on specified system geometry;

- RELAP5 model based on the preliminary design (3);

- Design Basis Event simulations;

- comparison between SPES3 and IRIS simulations;

- identification of the main differences and related reasons;

- update of the RELAP5 model and finalization of the SPES3

facility design to match the IRIS behavior $(4,5)$;

- Beyond Design Basis Event simulation.

The DBE simulations allowed to understand the plant behavior and the mutual interaction of the systems in transient conditions. The BDBE simulations were aimed at deepening specific safety aspects of the systems devoted to maintain the plant in safe conditions, in case of the most challenging accident occurrence.

The work summarized in this paper is aimed at describing how the design-simulation feedback process allowed to finalize the SPES3 facility design to simulate both DBE and BDBE with particular attention to the PCC intervention in case of EHRS unavailability.

\section{THE IRIS REACTOR AND THE SPES3 FACILITY}

The IRIS reactor integral layout is shown in Fig. 1. As all the main components are included in the reactor pressure vessel, the number of vessel penetrations is minimized together with the probability and size of LOCAs. Eight helical coil steam generators are located around the riser with eight spool pumps to circulate the primary fluid. The PRZ is included in the RPV dome and the control rod drive mechanisms are inside as well.

The IRIS containment and safety systems are sketched in Fig. 2. Two EBT are directly connected to the DVI line, high pressure side. Two stages of ADS helps the RPV depressurization in case of accident. Each of the secondary loops is provided with an EHRS rejecting the decay heat in the RWST when the reactor is isolated. The containment includes the DW and RC, the ADS QT, the PSS and the LGMS. A PCC system allows the plant long-term cooling in case of EHRS unavailability. The dynamic coupling of the primary and containment systems represents a unique feature in the accident mitigation strategy that allows to maintain the core covered even in the most challenging conditions.

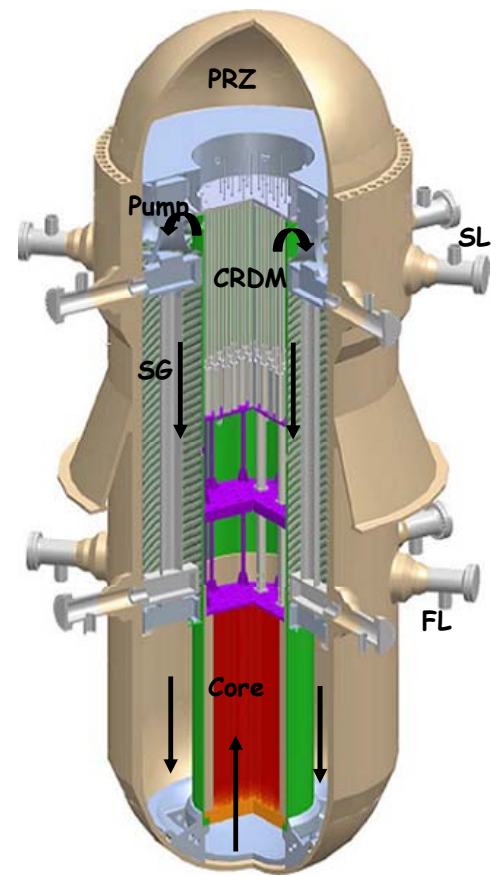

Fig. 1 IRIS integral layout

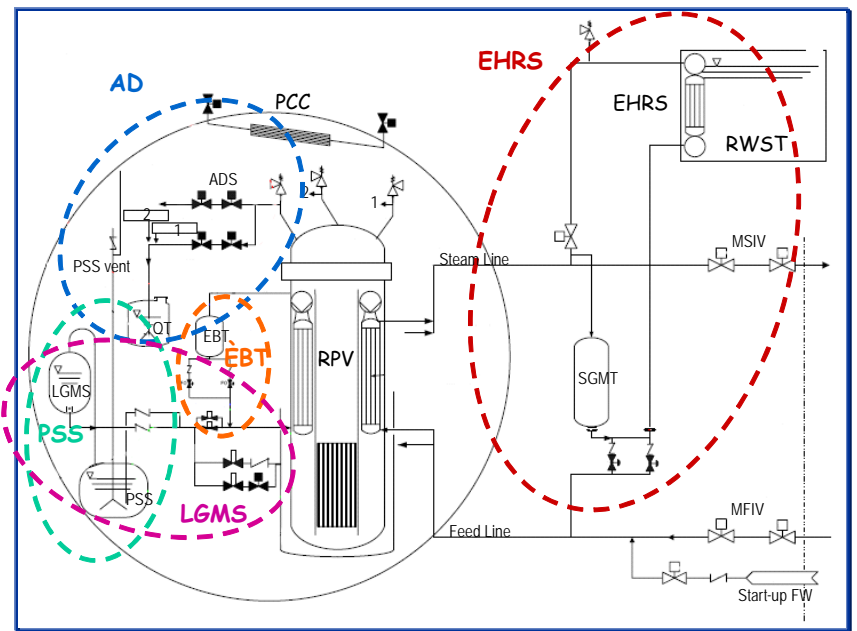

Fig. 2 IRIS containment and safety systems

The SPES3 facility is shown in Fig. 3. It reproduces the primary, secondary and containment systems of the reactor with 1:100 volume scale, full elevation and water at prototypical thermal-hydraulic conditions. The RPV includes the core simulator, the riser zone with CRDM in terms of occupied volume and pressure drops, the PRZ, the SGs, the annular 
downcomer and the lower plenum zone. The core is electrically heated with rods at prototypical geometry and pitch. The eight IRIS SGs are simulated by three helical coil SGs (representing 2, 2, 4 out of 8), wrapped around the central cylindrical riser. The four IRIS secondary loops are simulated, up to the main isolation valves, by three secondary loops (representing 1, 1, 2 of 4). Each SPES3 secondary loop is provided with an EHRS. Three heat exchangers, vertical tube type, are immersed in two RWSTs. The EHRS connected to the double secondary loop is in a pool, the other ones are in the other pool. One of the loops, with related containment tanks and safety systems, is shown in Fig. 4. The three IRIS ADS trains are simulated with two trains in SPES3, a single and a double one. The ADS Stage-I discharges into the QT and the Stage-II into the DW. The SPES3 PCC, U-tube horizontal bundle at the DW top, is not a representation of the prototypical IRIS PCC, but only dimensioned to remove the specified power. All the containment compartments are simulated with separate tanks connected by pipes. Where not existing in IRIS, great size pipes have been foreseen to limit the pressure drops; where existing, pressure drops are scaled by inserting proper orifices. Break line systems are designed to simulate split and DEG breaks of the DVI line, the EBT top line, the ADS Stage-I line, the FL and SL.

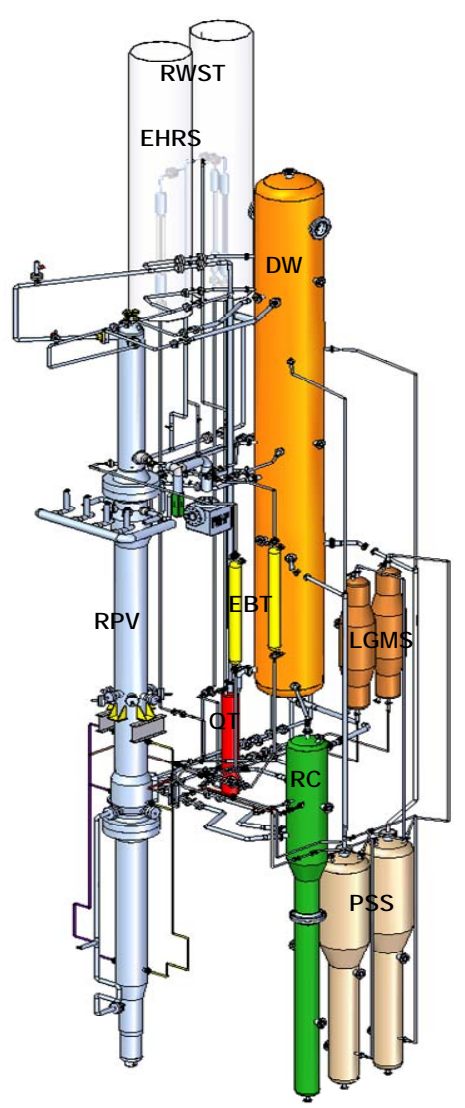

Fig. 3 SPES3 facility layout
The facility is suitable to perform both integral and separate effect tests, i.e to simulate $\mathrm{DBE}$ and $\mathrm{BDBE}$ and to verify the EHRS-SG heat transfer capabilities.

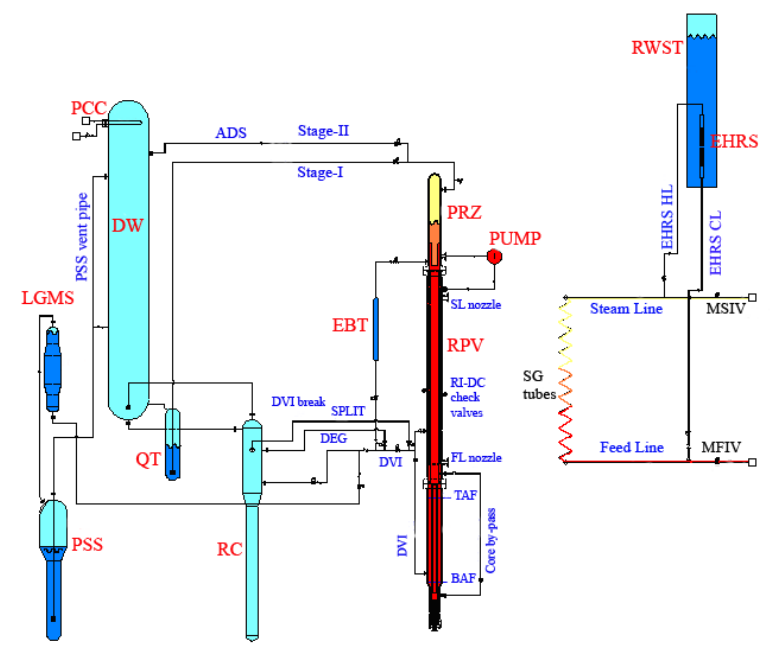

Fig. 4 SPES3 facility containment and safety systems

\section{NUMERICAL SIMULATION OF IRIS AND SPES3}

A complete model of the IRIS reactor was developed by FER of Zagreb University, for the RELAP5 and GOTHIC coupled codes. The plant model allowed to simulate the primary, secondary and EHRS systems with RELAP5 and the containment system with GOTHIC, Fig. 5 and Fig. 6. The whole SPES3 nodalization was developed for RELAP5, Fig. 7 and Fig. 8.

A simple direct explicit coupling of RELAP5/mod3.3 and GOTHIC $3.4 \mathrm{e}$ was used with connections at the points of hydraulic contact between vessel and containment (6). Both in the plant and facility nodalizations, the IRIS control system was simulated, limited to the signals needed for the simulation of specific transients.

A wide calculation-design feedback process led to optimize the SPES3 design to correctly simulate the IRIS reactor. The DBE DVI line DEG break was chosen as the reference transient for the investigation, being the most challenging LOCA foreseen in IRIS and potentially maximizing the RPV mass depletion.

The main transient phases and the system control logic, are shortly described in the followings: a) the break opening causes the RPV blowdown and depressurization, the containment pressurization, steam dumping into the PSS with air build-up at the PSS top and consequent pressurization; b) the S-signal (high DW pressure) triggers the reactor scram, the secondary loop isolation and the actuation of two EHRS; c) the low PRZ water level signal triggers the pump coastdown and the natural circulation in the core is guaranteed through the check valves connecting riser and downcomer at one-third of the SG height; d) the LM-signal (high DW pressure and low PRZ pressure) actuates the remaining EHRS, triggers the ADS Stage-I to help 
the RPV depressurization and the EBT intervention to inject cold borated water into the primary circuit; e) the low differential pressure signal between RPV and DW triggers the LGMS injection into the DVI line and opens the valves connecting $\mathrm{RC}$ and DVI line to increase the water reverse flow from the containment to the primary side; f) when the PSS pressurization is sufficiently high, cold water flows from PSS to DW, increasing the RC flooding and allowing water to reenter the RPV; g) the low LGMS mass signal opens the ADS stage-II connecting the primary and containment systems at high elevation in the plant with possible reverse steam flow from DW to RPV; h) in the long term, the plant is cooled by the EHRSs that reject the core decay heat to the RWST.

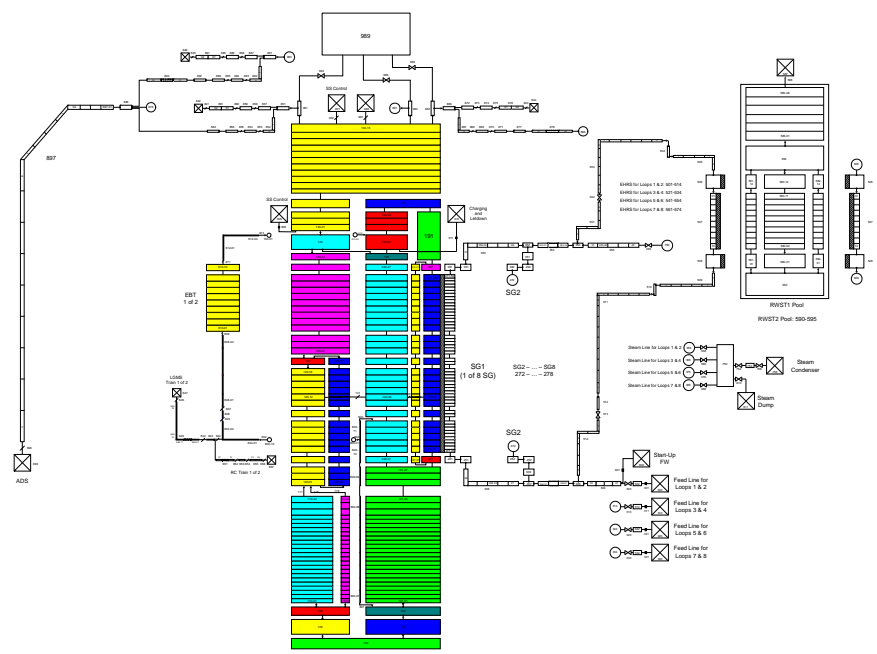

Fig. 5 IRIS loop nodalization for RELAP5 code
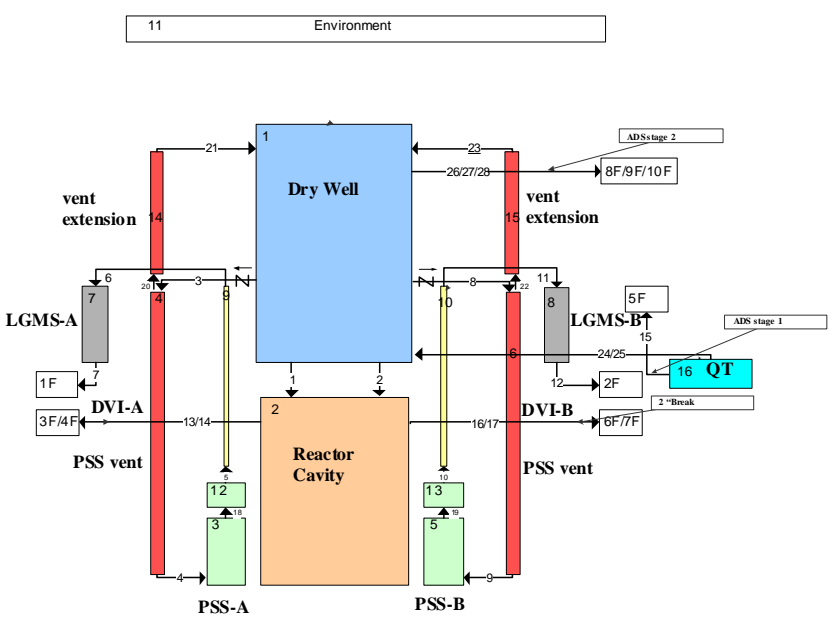

Fig. 6 IRIS containment nodalization for GOTHIC code
The comparison between the SPES3 and IRIS simulations allowed to modify step-by-step the SPES3 design up to the final configuration. Sensitivity cases were run to investigate specific aspects affecting the trend of the main parameters of the plant. The containment heat structures, the heat transfer from EHRS to RWST and the piping pressure drops were found to be the most affecting parameters in matching the IRIS results. Particular attention was addressed to the containment pressure trend, very important in the primary and containment system dynamic coupling, during the accident, and affecting the water transfer toward the RPV, fundamental to maintain the core covered and the fuel clad temperature at limited values (5).

Fig. 9, Fig. 10 and Fig. 11 show the IRIS and SPES3 trend of the most meaningful quantities, evidencing a good agreement in the containment peak pressure and long-term values, similar RPV mass and no heater rod clad temperature excursions, even at the minimum primary mass inventory. The stronger IRIS containment depressurization, after the pressure peak, is mainly due to the anticipated PSS to DW water transfer and LGMS faster emptying in IRIS, Fig. 12, Fig. 13.

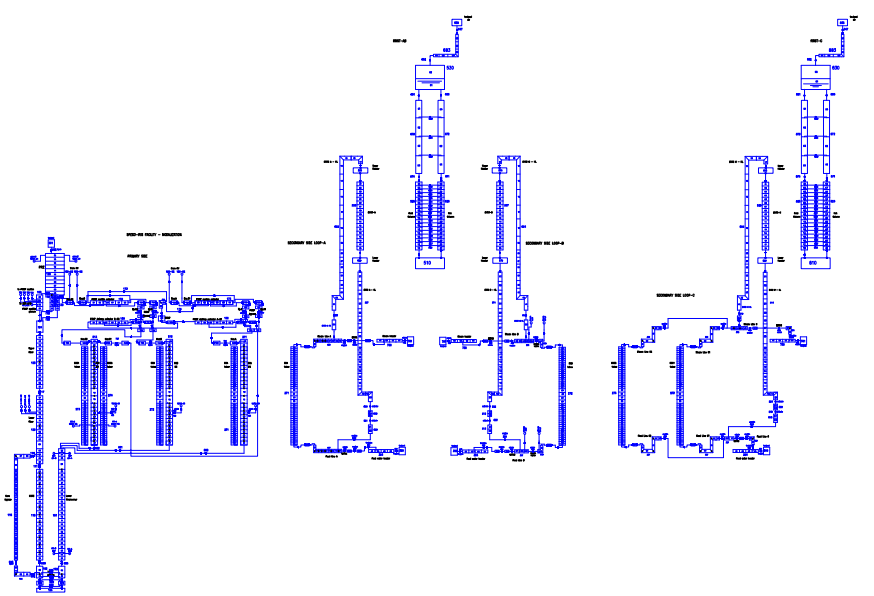

Fig. 7 SPES3 loop nodalization for RELAP5 code

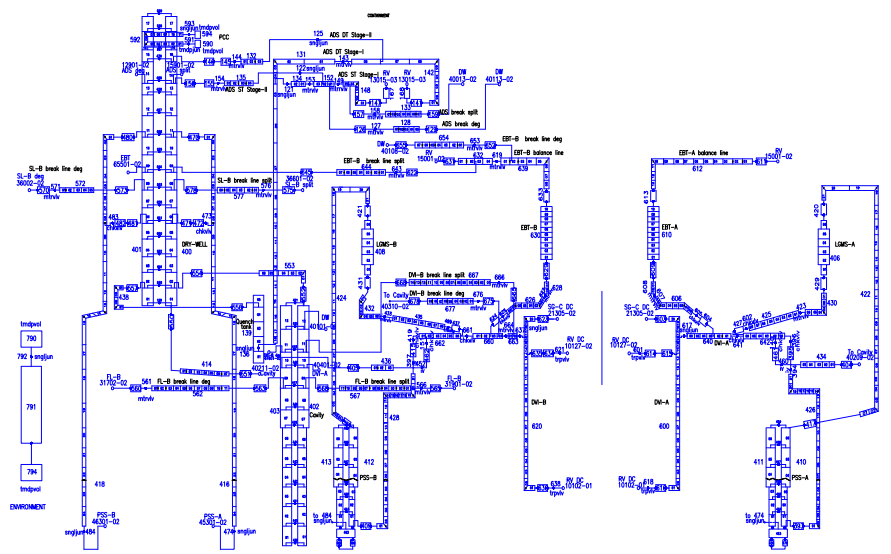

Fig. 8 SPES3 containment nodalization for RELAP5 code 


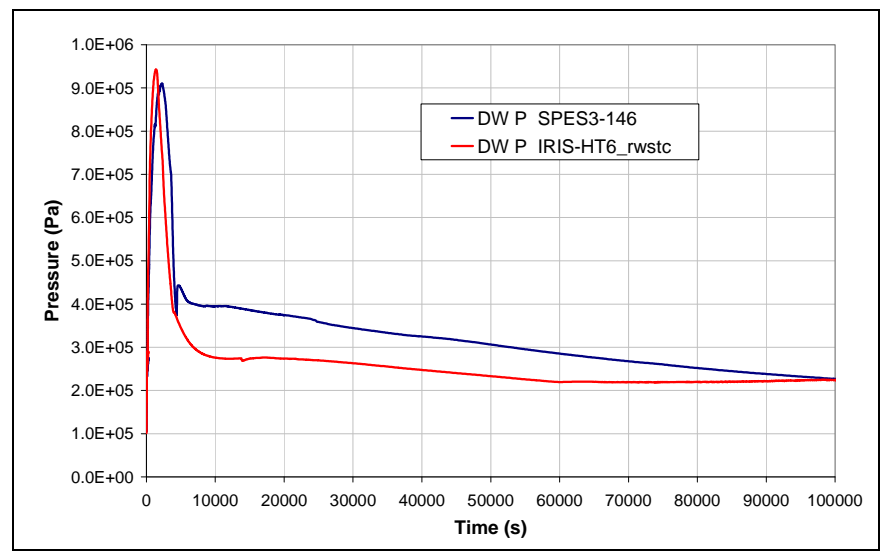

Fig. 9 DBE DVI line DEG break: DW pressure

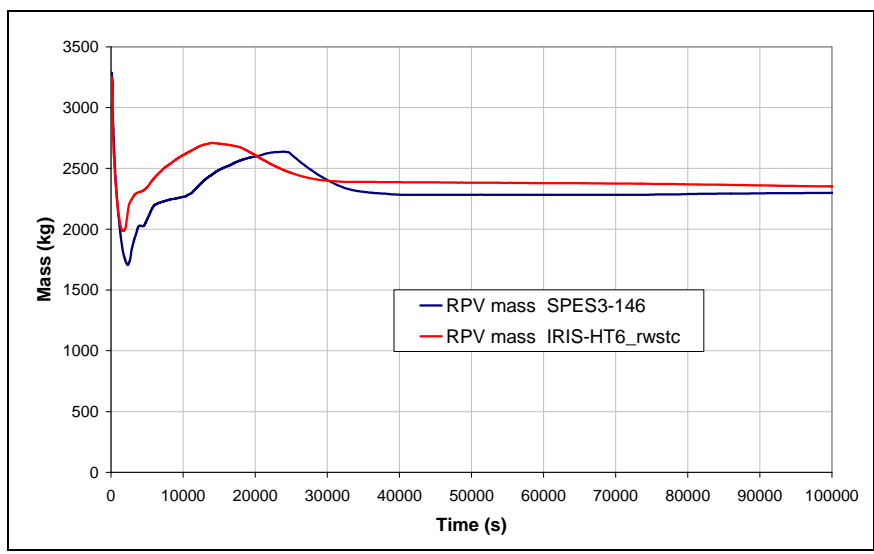

Fig. 10 DBE DVI line DEG break: RPV mass

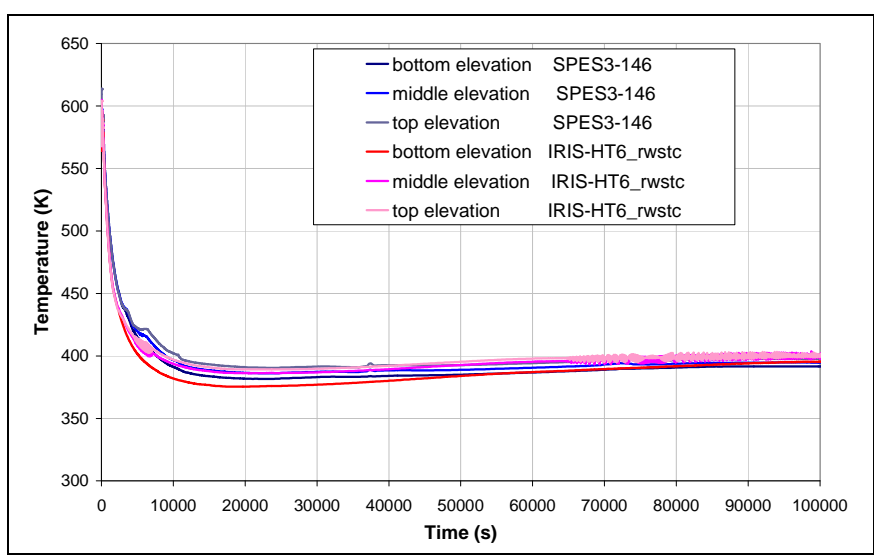

Fig. 11 DBE DVI line DEG break: core rod clad temp.

In the phase of long-term cooling, the decay heat is removed by the EHRS. The water back-flow from the RC to the RPV contributes to maintain the core covered and enhances the natural circulation in the core, Fig. 14, mainly driven by the water gravity head in the containment, above the RPV level.

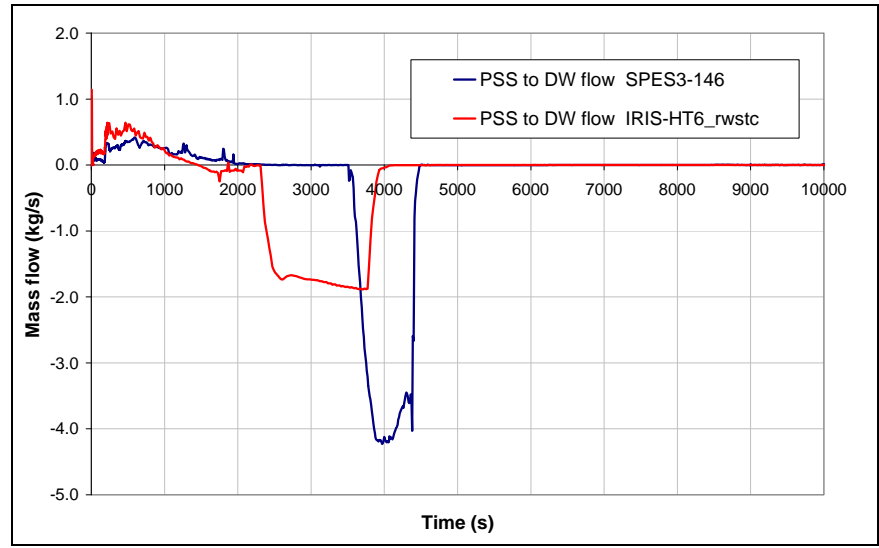

Fig. 12 DBE DVI line DEG break: DW to PSS mass flow

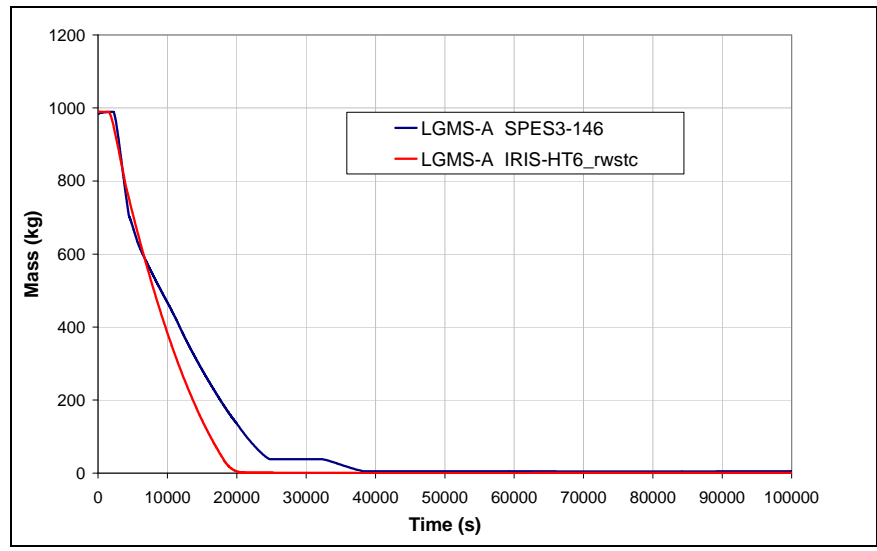

Fig. 13 DBE DVI line DEG break: IL-LGMS mass

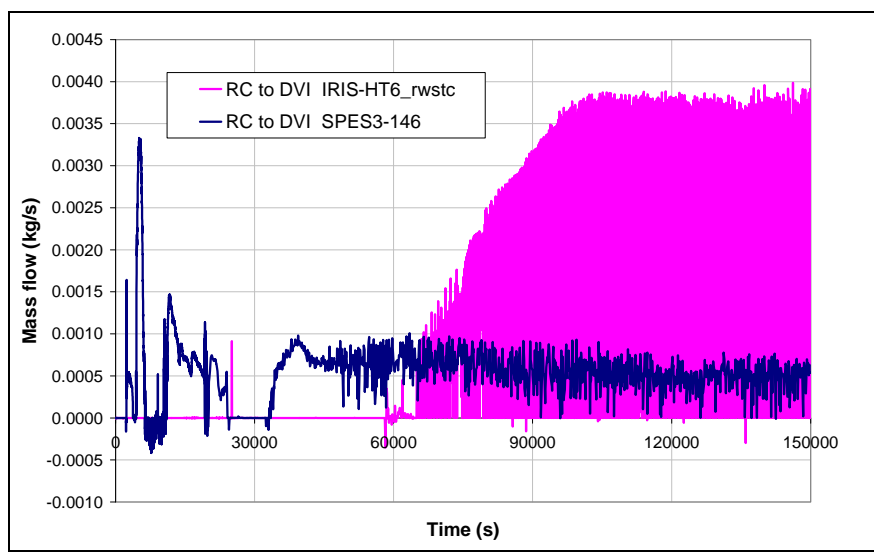

Fig. 14 DBE DVI line DEG break: RC to IL-DVI line mass flow

\section{SIMULATION OF THE BDBE DVI LINE DEG BREAK}

Once optimized the SPES3 design and nodalization on the basis of the 2-inch equivalent DBE DVI line DEG break, the SPES3 model was tested against the BDBE DVI line DEG break, where all EHRSs are unavailable and the PCC is the 
only device suitable to remove the decay heat. The SPES3 PCC removes heat by a water flow in change of phase, tube side, that condenses the DW steam and maintains the containment pressure between 0.8 and $0.9 \mathrm{MPa}$. The simulation of such BDBE allowed to verify the PCC performance and to give indications to optimize the accident mitigation strategies, on the basis of the PCC and ADS Stage-II actuation time. The SPES3 steady conditions, before the break, are reported in Tab. 1 .

\section{Tab. 1 SPES3 initial and boundary conditions}

\begin{tabular}{|l|c|c|}
\hline \multicolumn{1}{|c|}{ SPES3 } & Unit & Value \\
\hline Pressurizer pressure & $\mathrm{MPa}$ & 15.55 \\
\hline Core power & $\mathrm{MW}$ & 10 \\
\hline Core inlet fluid temperature & $\mathrm{K}$ & 565.7 \\
\hline Core outlet fluid temperature & $\mathrm{K}$ & 603.1 \\
\hline Primary total mass flow & $\mathrm{kg} / \mathrm{s}$ & 47.8 \\
\hline SG outlet pressure & $\mathrm{MPa}$ & 5.83 \\
\hline SG feed water temperature & $\mathrm{K}$ & 497.1 \\
\hline SG-A steam outlet temperature & $\mathrm{K}$ & 591.5 \\
\hline SG-B steam outlet temperature & $\mathrm{K}$ & 588.1 \\
\hline SG-C steam outlet temperature & $\mathrm{K}$ & 588.1 \\
\hline SG-A mass flow & $\mathrm{kg} / \mathrm{s}$ & 1.25 \\
\hline SG-B mass flow & $\mathrm{kg} / \mathrm{s}$ & 1.25 \\
\hline SG-C mass flow & $\mathrm{kg} / \mathrm{s}$ & 2.50 \\
\hline Containment pressure & $\mathrm{MPa}$ & 0.1013 \\
\hline Containment water temperature & $\mathrm{K}$ & 322.1 \\
\hline RWST fluid temperature & $\mathrm{K}$ & 298 \\
\hline
\end{tabular}

The simulated BDBE foresees the failure of the EHRS-A and $\mathrm{B}$ actuation on S-signal and the failure of the EHRS-C on LM-signal. The PCC is actuated with $1800 \mathrm{~s}$ delay on LMsignal, assuming such delay as the time required to fill-up the containment refueling cavity that provides a heat sink for the passive containment cooling system.

The main phases of the transient are shortly described in the followings: a) the break opening causes the RPV blowdown and depressurization, the containment pressurization and steam dumping into the PSS with air build-up at the PSS top; b) the $\mathrm{S}$-signal triggers the reactor scram and the secondary loop isolation. The EHRS-A and B actuation fails; c) the low PRZ water level signal triggers the pump coastdown and the natural circulation in the core is guaranteed until the RPV water level is above the check valves connecting riser and downcomer; d) the LM-signal, triggers the ADS Stage-I to help the RPV depressurization and the EBT intervention to inject cold borated water into the primary circuit. The EHRS-C actuation fails; e) the PCC water flow is actuated when the containment pressure threshold reaches $0.9 \mathrm{MPa}$ and after $1800 \mathrm{~s}$ since the LM-signal; f) the PCC depressurizes the containment and, when the PSS pressure is sufficiently high to win the gravity head of the PSS vent pipes, cold water flows from PSS to DW increasing the containment depressurization and the RC flooding; g) the low differential pressure signal between RPV and DW triggers the LGMS injection into the DVI line and opens the valves connecting RC and DVI line to increase the water back-flow from the containment to the primary system; h) the PCC maintains the DW pressure between the specified set points; i) the low LGMS mass signal $(20 \%$ of the initial mass) opens the ADS Stage-II. The PRZ and DW pressures equalize and water flows from RC to RPV driven by the containment water gravity head; 1) in the long term, the PCC maintains the system at limited pressure values by condensing steam exiting the RPV, with a water back-flow from containment to primary.

The above described phases refer to the SPES3-160 case, as reported in Tab. 2. Such case showed some criticalities in the transient evolution with a high containment pressure peak (1.35 $\mathrm{MPa}$ ) and a long period of low RPV mass inventory ( 35000 s) with consequent core uncovering and high rod clad temperature (up to $870 \mathrm{~K}$ ).

The search of the causes for such criticalities led to study how the PCC actuation delay on LM-signal and the ADS StageII opening time affects the transient. The sensitivity cases SPES3-159, 162 and 158, were run as reported in Tab. 2. The anticipation of the PCC actuation time ( 0 and $1000 \mathrm{~s}$ delay on LM-signal) led to reduce the containment pressure peak. The ADS Stage-II opening anticipation led to an earlier DW and RPV pressure equalization, with the consequent enhancement of the RC to RPV water flow, through the DVI line.

Tab. 2 BDBE DVI line DEG break sensitivity cases

\begin{tabular}{|c|c|c|c|}
\hline Case & $\begin{array}{c}\text { PCC actuation } \\
\text { delay on LM }\end{array}$ & $\begin{array}{c}\text { ADS Stage-II } \\
\text { actuation on low } \\
\text { LGMS mass }\end{array}$ & $\begin{array}{c}\text { RC to DVI } \\
\text { orifice }\end{array}$ \\
\hline SPES3-160 & $1800 \mathrm{~s}$ & $20 \%$ & $1 \mathrm{~mm}$ \\
\hline SPES3-159 & $0 \mathrm{~s}$ & $20 \%$ & $1 \mathrm{~mm}$ \\
\hline SPES3-162 & $1800 \mathrm{~s}$ & $80 \%$ & $1 \mathrm{~mm}$ \\
\hline SPES3-158 & $1000 \mathrm{~s}$ & $20 \%$ & $1 \mathrm{~mm}$ \\
\hline & & & \\
\hline SPES3-163 & $1800 \mathrm{~s}$ & $20 \%$ & $6 \mathrm{~mm}$ \\
\hline SPES3-164 & $0 \mathrm{~s}$ & $20 \%$ & $6 \mathrm{~mm}$ \\
\hline SPES3-165 & $1800 \mathrm{~s}$ & $80 \%$ & $6 \mathrm{~mm}$ \\
\hline SPES3-166 & $1000 \mathrm{~s}$ & $20 \%$ & $6 \mathrm{~mm}$ \\
\hline
\end{tabular}

The DW pressure is shown in Fig. 15. The cases SPES3160 and 162, show the same high pressure peak and cycling PCC operation. The cases SPES3-159 and 158 show more limited pressure peaks and the same cycling PCC operation. On the other hand, the reduction of the containment pressurization limits the PSS pressurization with the negative consequence of the lack or insufficient back-flow from PSS to DW and the negative effect of lower RPV mass make-up. Fig. 16 shows the total DW to PSS mass flow: in the first phase, the steam-air mixture flows from the DW to the PSS and in the second phase water flows from PSS to DW. Due to the anticipated PCC intervention, the mixture transfer in cases SPES3-159 and 158 is lower than in cases SPES3-160 and 162 and the PSS pressurization is not enough to overcome the PSS vent line 
gravity head. That prevents the back-flow from PSS to DW in case SPES3-159 and limits it in SPES3-158. The water transfer from the containment to the primary system occurs by the LGMS and the RC, through the DVI line, when the containment pressure overcomes the RPV pressure. Fig. 17 shows the intact loop LGMS mass and Fig. 18 shows the mass flow from the RC to the RPV through the intact DVI line. The anticipation of the ADS Stage-II opening, based on the low LGMS mass signal, allows an earlier pressure balance between the primary and containment systems, enhancing the RPV water inlet both from LGMS and RC with consequent mass inventory recovery, Fig. 17, Fig. 18, Fig. 19.

Fig. 20 reports the core rod clad temperature at high elevation. All the cases show high temperature excursions and only in SPES3-162 the core is definitively rewetted in about $6000 \mathrm{~s}$, thanks to the injection of water from the RC.

The SPES3 core decay power in the long term is around 50 $\mathrm{kW}$ and the mass flow needed to remove it by evaporation is about $0.02 \mathrm{~kg} / \mathrm{s}$. Fig. 18 shows that, water entering the RPV by the $\mathrm{RC}$, is about one tenth of the required one.

The solution of increasing the RC to DVI line orifice diameter was adopted passing from $1 \mathrm{~mm}$ to $6 \mathrm{~mm}$, to guarantee the required mass flow under an estimated differential pressure of about $200 \mathrm{~Pa}$, suitable to overcome the local and distributed pressure drops between RC and DVI line.

Further four sensitivity cases were run, as reported in Tab. 2 , with the same characteristics as the cases above described. The containment pressure trend, shown in Fig. 21, is similar to that in Fig. 15. The cases SPES3-163 and 165, show the same high pressure peak and cycling PCC operation.

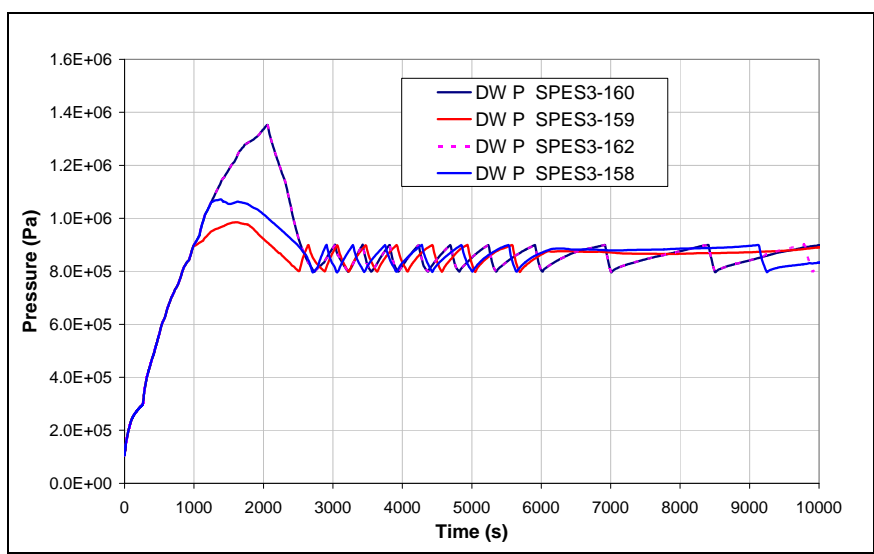

Fig. 15 BDBE DVI line DEG break: DW pressure

The cases SPES3-164 and 166 show a more limited pressure peak and the same cycling PCC operation. As for cases SPES3159 and 158, the lower containment pressurization limits the PSS pressurization with the negative consequence of insufficient back-flow from PSS to DW and lower RPV mass make-up.

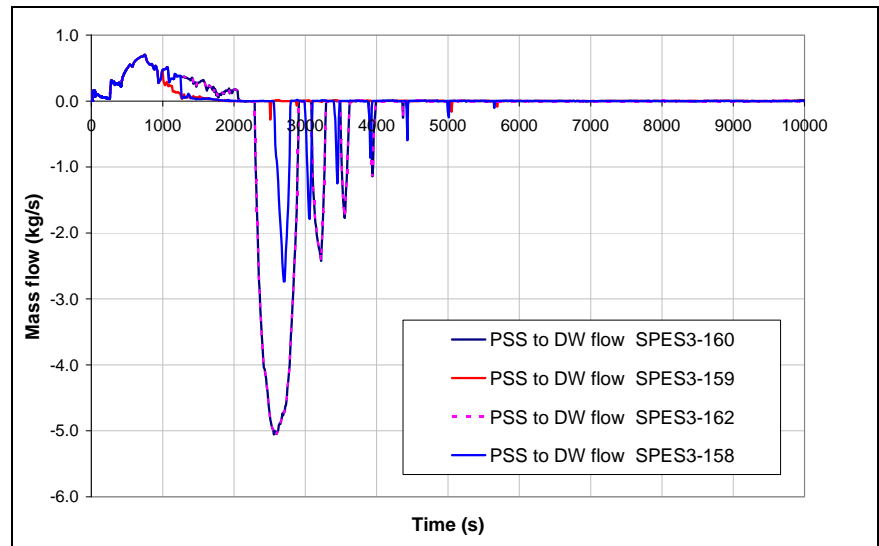

Fig. 16 BDBE DVI line DEG break: DW to PSS mass flow

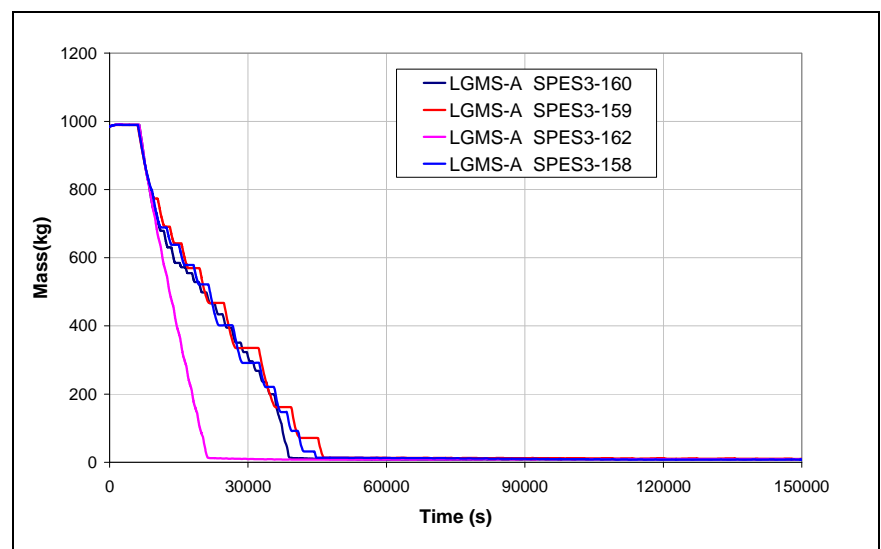

Fig. 17 BDBE DVI line DEG break: IL-LGMS mass

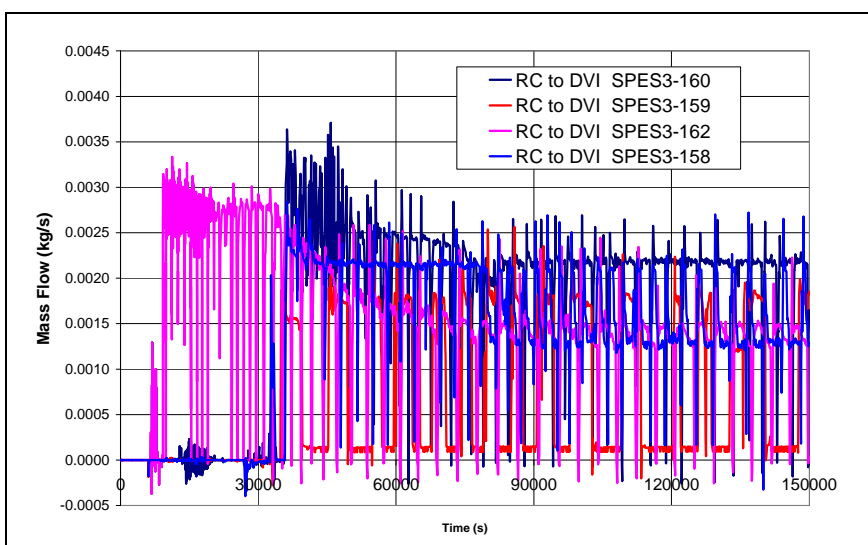

Fig. 18 BDBE DVI line DEG break: RC to IL-DVI line mass flow

Fig. 22 shows the total DW to PSS mass flow that, in the first phase, directs the steam-air mixture from the DW to the PSS. Due to the anticipated PCC intervention, in SPES3-164 and 166, such mixture transfer is lower than in cases SPES3163 and 165 and the PSS pressurization is not enough to overcome the PSS vent line gravity head. Similarly to cases SPES3-159 and 158, that almost prevents the back-flow from 
PSS to DW in case SPES3-164 and limits it in SPES3-166. The water transfer from containment to primary system by the LGMS and the RC, through the DVI line, occurs when the containment pressure overcomes the RPV pressure. Fig. 23 shows the intact loop LGMS mass and Fig. 24 reports the mass flow from the RC to the RPV through the intact DVI line. The anticipation of the ADS Stage-II opening, based on the low LGMS mass signal, allows an earlier pressure balance between primary and containment systems, enhancing the RPV water inlet both from LGMS and RC with consequent mass inventory recovery, Fig. 23, Fig. 24, Fig. 25. The comparison between Fig. 24 and Fig. 18 put in evidence that the RC to DVI line orifice enlargement allows to increase the RPV entering mass flow up to values suitable to remove the decay power.

Fig. 26 reports the core rod clad temperatures at high elevation. All the cases show high temperature excursions and in case SPES3-165, as in case SPES3-162, the core is rewetted in about $6000 \mathrm{~s}$. Thanks to the enhanced injection of water from the RC, also the other cases are definitively rewetted after longer times, up to $50000 \mathrm{~s}$, even if undergoing very high clad temperatures, up to $1140 \mathrm{~K}$ in case of SPES3-164. The improvement in the accident mitigation is clearly reached by enhancing the water transfer from the RC to the RPV.

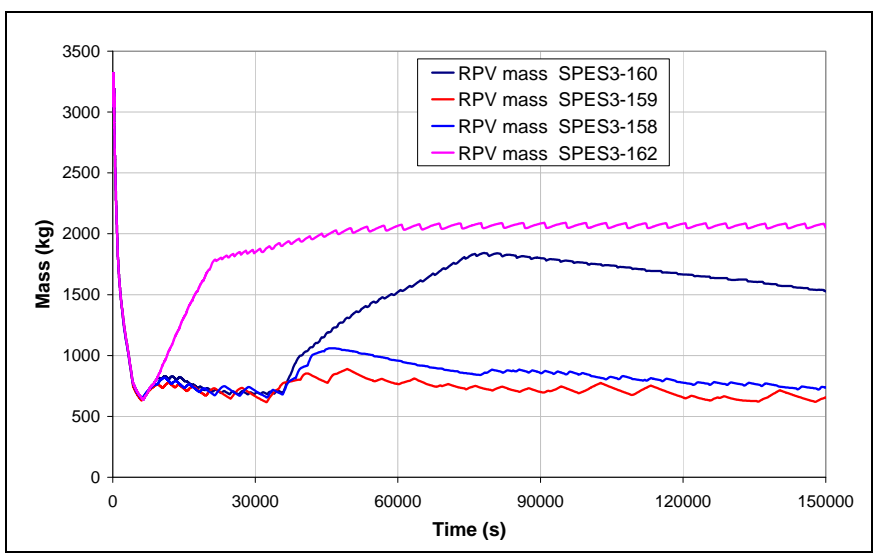

Fig. 19 BDBE DVI line DEG break: RPV mass

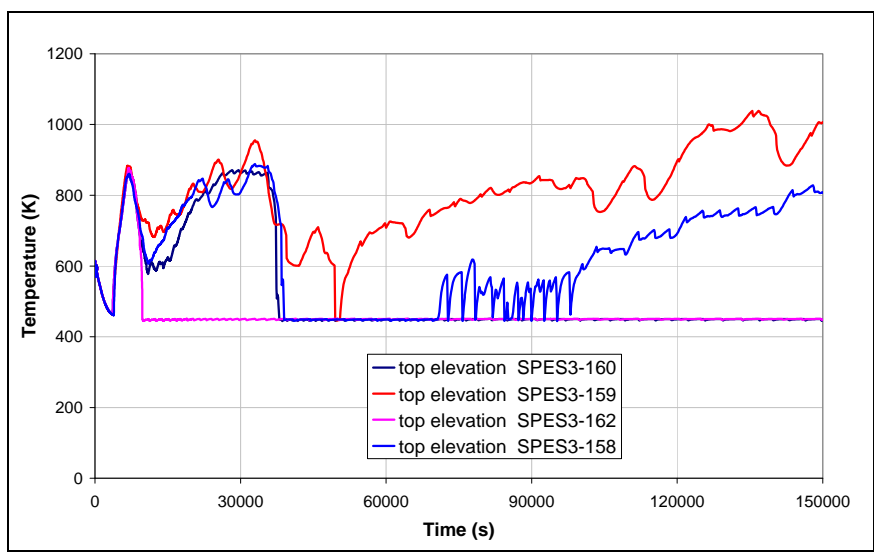

Fig. 20 BDBE DVI line DEG break: core rod clad temp.

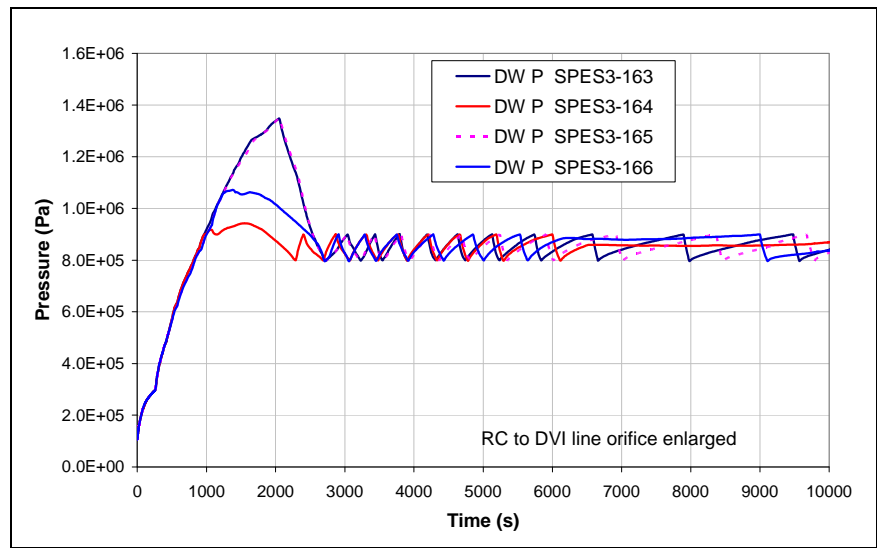

Fig. 21 BDBE DVI line DEG break: DW pressure

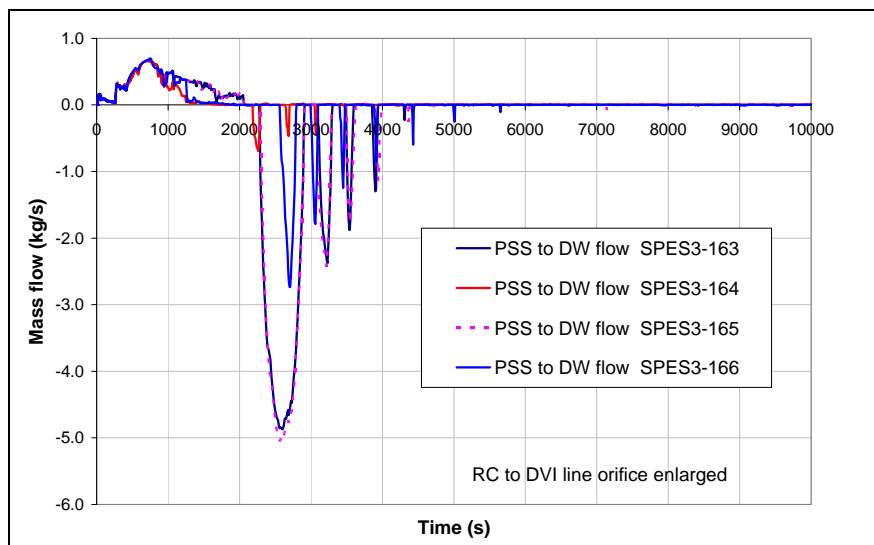

Fig. 22 BDBE DVI line DEG break: DW to PSS mass flow

\section{ACHIEVEMENTS}

The RELAP5 sensitivity analyses of the SPES3 facility BDBE DVI line DEG break led to understand more in depth the PCC operation influence on the accident mitigation.

Moreover, the importance of the ADS Stage-II opening was evidenced by the water back-flow enhancement from the containment to the primary system, by equalizing RPV and DW pressure and reducing the primary side resistance to the water inlet.

The sensitivity cases showed also a limit in the facility design, related to the too narrow orifice on the RC to DVI line, which must be properly sized to provide the necessary water mass flow from containment to primary system to remove the decay power. The simulation of the DBE did not evidence such limit, as the EHRS removed the decay heat from the primary side in natural circulation through the riser to downcomer check valves (5).

According to the BDBE simulation results, the PCC actuation with $1800 \mathrm{~s}$ delay on the LM-signal seems to be the optimum to have the needed PSS pressurization and a still acceptable containment pressure peak. 


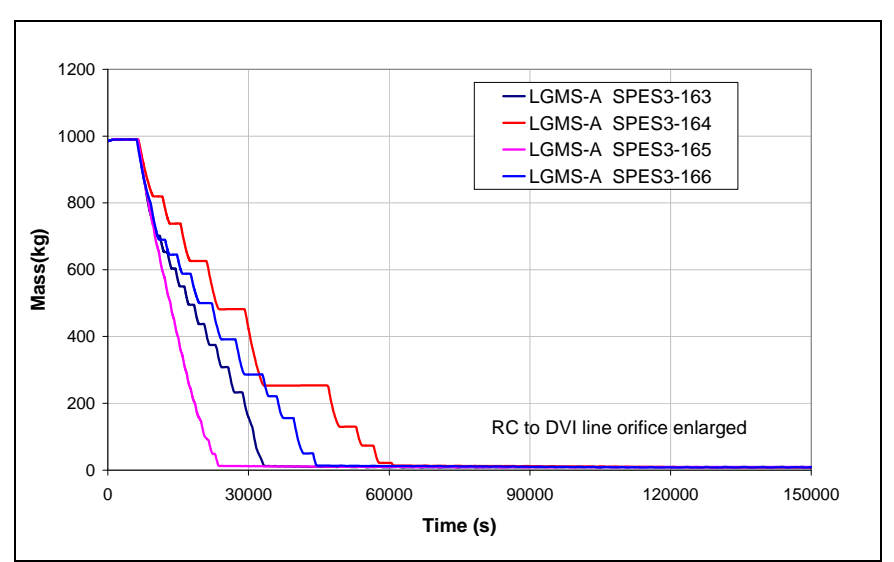

Fig. 23 BDBE DVI line DEG break: IL-LGMS mass

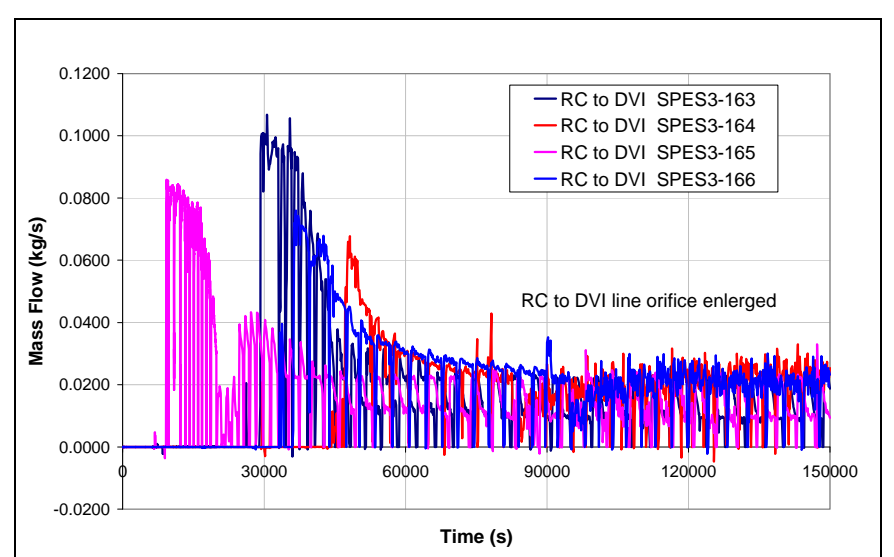

Fig. 24 BDBE DVI line DEG break: RC to IL-DVI line mass flow

The PCC actuation anticipation is not a good solution in the accident management as, preventing the PSS to DW backflow, reduces the water amount available to be injected into the RPV for mass inventory recovery.

The ADS Stage-II actuation anticipation seems fundamental to equalize primary and containment pressure as soon as possible to enhance the water back-flow toward the RPV. Even in this case, the DBE simulation had not evidenced criticalities linked to this event, as, once made-up the RPV mass inventory, the EHRSs remove the decay heat with no need of a large mass transfer between RPV and containment. The ADS Stage-II actuation signal, originally based on the reaching of the $20 \%$ LGMS initial mass, was conditioned to the reaching of the $80 \%$ LGMS initial mass. A further anticipation would allow an earlier core covering and rod clad temperature excursion limitation in the BDBE. The ADS Stage-II will be actuated on different signals in case of DBE or BDBE.

The RC to DVI line orifice diameter was optimized passing from $1 \mathrm{~mm}$ to $6 \mathrm{~mm}$.

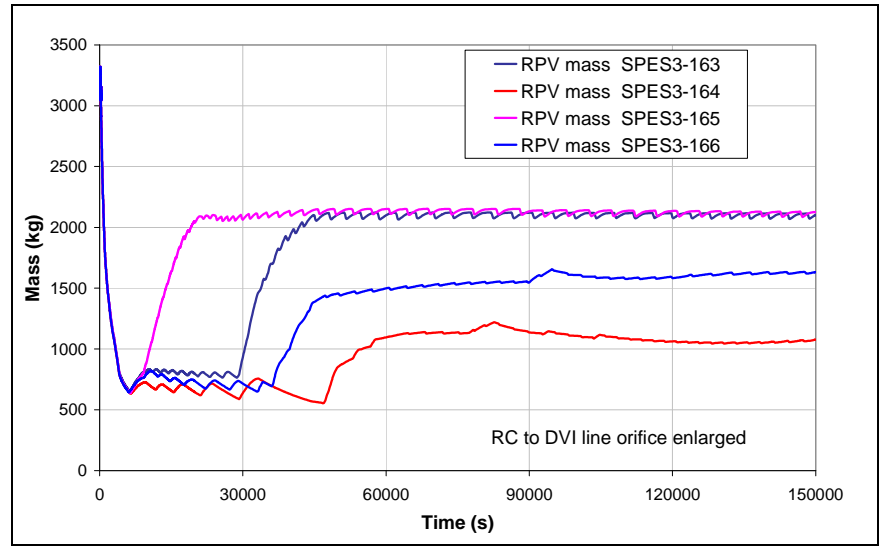

Fig. 25 BDBE DVI line DEG break: RPV mass

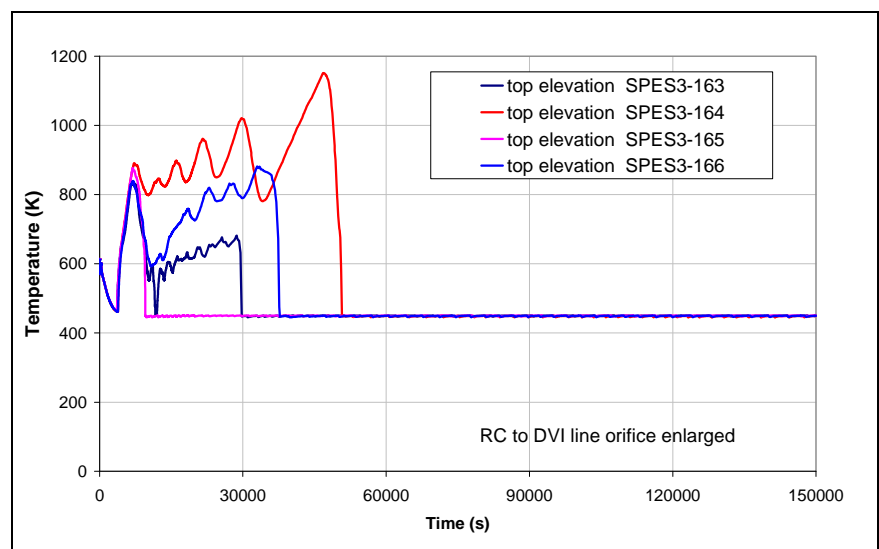

Fig. 26 BDBE DVI line DEG break: core rod clad temp.

\section{CONCLUSIONS}

The BDBE DVI line DEG break simulation on the SPES3 facility led to better understand problems and criticalities in the intervention of the safety systems devoted to maintain the plant in safe conditions, even with all the EHRS lost. The results evidenced the importance of the PCC and ADS Stage-II actuation time in the transient evolution and core cooling. They provided fundamental indications on the accident mitigation strategies suitable to let the plant survive even under the most severe accident conditions. The actuation time optimization of the safety system was performed and it will be introduced in the SPES3 facility test procedures. The optimization of the geometric parameter on the RC to DVI line will allow to update the final SPES3 design.

In the future, the simulations of other test matrix BDBEs will provide information for a deeper phenomena investigation and further optimization of the system. 


\section{NOMENCLATURE}

$\begin{array}{ll}\text { ADS } & \text { Automatic Depressurization System } \\ \text { BAF } & \text { Bottom of Active Fuel } \\ \text { BDBE } & \text { Beyond Design Basis Events } \\ \text { CRDM } & \text { Control Rod Drive Mechanism } \\ \text { DBE } & \text { Design Basis Events } \\ \text { DC } & \text { Downcomer } \\ \text { DEG } & \text { Double Ended Guillotine } \\ \text { DVI } & \text { Direct Vessel Injection } \\ \text { DW } & \text { Dry Well } \\ \text { EBT } & \text { Emergency Boration Tanks } \\ \text { EHRS } & \text { Emergency Heat Removal System }\end{array}$

ENEA Agenzia nazionale per le nuove tecnologie, l'energia e lo sviluppo economico sostenibile (national agency for new technologies, Energy and sustainable development)

EPZ Emergency Planning Zone

FL Feed Line

FW Feed Water

GNEP Global Nuclear Energy Partnership

GOTHIC Generation Of Thermal-Hydraulic Information for Containments

I\&C Instrumentation and Control

IL Intact Loop

IRIS International Reactor Innovative and Secure.

ITF Integral Test Facility

LGMS Long Term Gravity Make-up System

LOCA Loss of Coolant Accident

LM Loca Mitigation

LWR Light Water Reactor

MFIV Main Feed Isolation Valve

MSIV Main Steam Isolation Valve

NPP Nuclear Power Plant

PCC Passive Containment Condenser

PRZ Pressurizer

QT Quench Tank

RC Reactor Cavity

RELAP Reactor Excursion and Leak Analysis Program

RI Riser

RPV Reactor Pressure Vessel

RWST Refueling Water Storage Tank

S Safeguard

SG Steam Generator

SGMT Steam Generator Make-up Tank

SIET Società Informazioni Esperienze Termoidrauliche (Company Information and Experiences on Thermalhydraulics

SPES3 Simulatore Pressurizzato per Esperienze di Sicurezza (Pressurized Simulator for Safety Tests)

ST Steam Line

TAF Top of Active Fuel

temp. temperature

\section{REFERENCES}

[1] Carelli M. D., et al, 2004, "The Design and Safety Features of the IRIS Reactor," Nuclear Engineering and Design, 230, pp. 151-167.

[2] M. D. Carelli, et al, 2005, "IRIS design overview and status update". ICONE13-50442 Beijing, China. May 16-20.

[3] M. Carelli, et al, 2009, "The SPES3 experimental facility design for the IRIS Reactor simulation", Science and Technology of Nuclear Installations. Volume 2009, Article ID 579430.

[4] R. Ferri, et al, 2010, "SPES3 facility and IRIS reactor numerical simulations for the SPES3 final design", ENC 2010 European Nuclear Conference. Barcelona, Spain, May $30-$ June 2.

[5] A. Achilli, et al, 2011, "SPES3 facility RELAP5 sensitivity analyses on the containment system for design review", Science and Technology of Nuclear Installations, Special Issue "Integral Test Facilities and Thermal-Hydraulic System Codes in Nuclear Safety Analysis".

[6] D. Grgić, et al, 2003, "Coupled RELAP5 / GOTHIC Model for IRIS Reactor SBLOCA Analysis”. ICAPP 2003 Córdoba, Spain, May 4-7. 